\title{
Inventario y problemas de conservación de las huellas de dinosaurio en Marruecos
}

El aumento de estudios y publicaciones pone de manifiesto el valor natural, cultural y científico de las huellas de dinosaurio de Marruecos. Más de 3.000 huellas inventariadas y un número desconocido en los yacimientos todavía inexplorados componen la riqueza patrimonial del país norteafricano, con más de 27 lugares con huellas ubicados mayoritariamente en la cordillera del Atlas. A pesar de la constancia de la riqueza patrimonial, los yacimientos se ven sometidos a problemas de conservación como la meteorización, la explotación de canteras o la descoordinación entre administraciones.

Félix Pérez-Lorente | Dpto. de Química, Universidad de La Rioja

Mohamed Boutakiout | Facultad de Ciencias, Universidad Mohamed V de Rabat

Moussa Masrour | Universidad Ibn Zohr de Agadir

URL de la contribución <www.iaph.es/revistaph/index.php/revistaph/article/view/4143>

Las huellas fósiles de dinosaurio son un bien paleontológico, de interés científico y cultural cuyo valor patrimonial se puede cuantificar con los datos que exponemos. Los lugares con huellas de dinosaurio en Marruecos se extienden, sobre todo, por la cordillera del Atlas. Hay veintisiete lugares con huellas que engloban treinta y seis yacimientos. Su extensión varía desde unos cientos de metros cuadrados (Talmest, $200 \mathrm{~m}^{2}$ ) a más de $56.000 \mathrm{~m}^{2}$ (louaridène). Algunos contienen un solo afloramiento (como Aït Blal, Talmest), pero otros tienen varios afloramientos en su interior (es el caso de Aït Bou Guemez, Imilchil, El Mers...). La distancia entre algunos afloramientos es del orden del kilómetro (Aït Bou Guemez, Ibaqalliwn, Aguer n Ouzrou, Tabant, Ibaqalliwn, Ahbak, Sidi Moussa); en otros, de menos de 10 metros (louaridène). El inventario de lugares y yacimientos cambiará porque quedan lugares inexplorados (como El Mers).

El total de huellas, según los datos de yacimientos completos (Jarrate Lbel, Anza, Talmest, Ifgh, Aït Blal, Arghbalou-Taoudaat, Adrar-n-Ougladal) y yacimientos parciales (Imilchil, louaridène, El Mers, Bin el Ouidane, Aït Bou Guemez y Ait Arbi), es de 2.253 huellas terópodas, 33 ornitisquias, 998 saurópodas, 102 prosaurópodas, 11 pterosaurias y 102 de cocodrilos. No se sabe cuántas huellas hay en los demás yacimientos conocidos

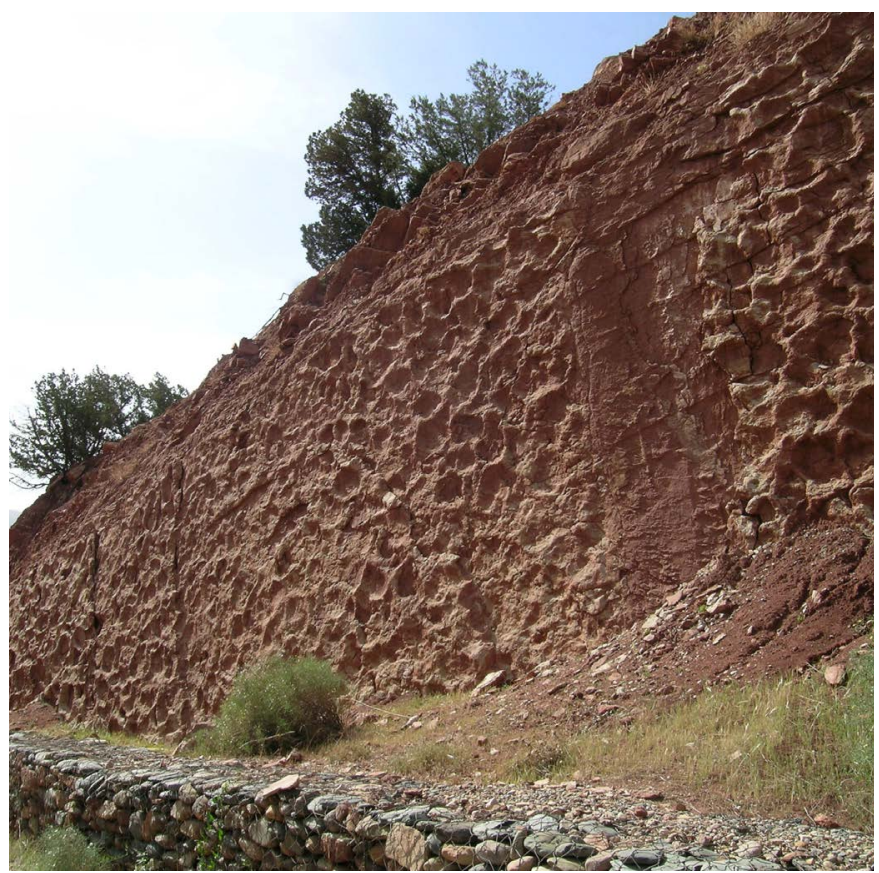

Aspecto parcial del yacimiento de Imi-n-Tanout | foto Félix Pérez-Lorente

ni los cientos de pisadas de la pared de más de $150 \mathrm{~m}$ de largo de Imi-n-Tanout.

Las huellas marroquíes (ciertas huellas de pterosaurios se supusieron de dinosaurios) representan la mayor parte de los grupos taxonómicos de dinosaurios (inclui- 


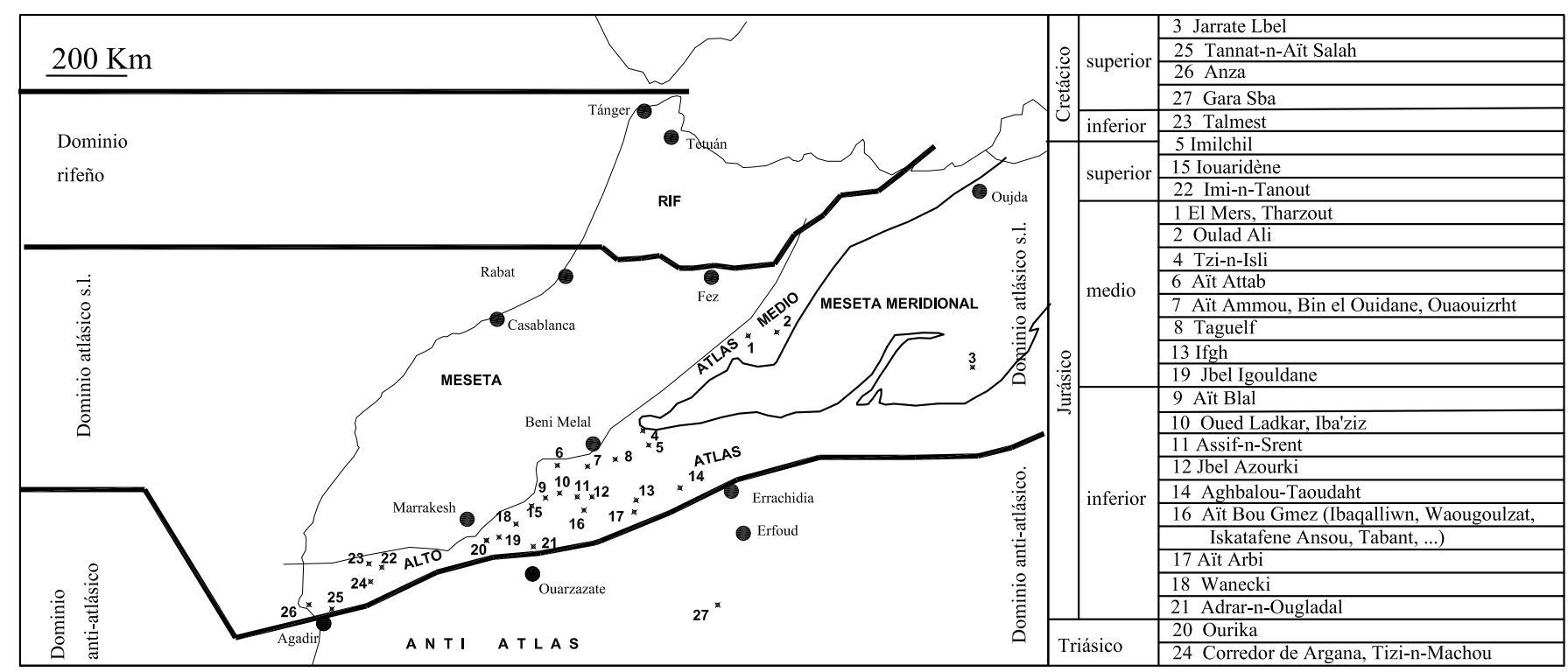

Distribución de los 27 lugares con huellas de dinosaurio descubiertos en Marruecos | gráfico Pérez-Lorente, Boutakiout, Masrour

das las aves). Pterosaurios, cocodrilos y dinosaurios (Arcosauria) y tortugas (Anapsida) dejaron sus huellas:

1. Dinosaurios:

$\begin{array}{lll}\text { Otozoum } & \text { Breviparopus } & \text { Parabrontopodus } \\ \text { Iguanodontipus } & \text { Deltapodus } & \text { Anomoepus } \\ \text { Boutakioutichniun } & \text { Bresaniochnus } & \text { Carmelopodus } \\ \text { Changpeipus } & \text { Deferrariichnium } & \text { Dineichnus } \\ \text { Macropodosaurus } & \text { Megalosaurus } & \text { Megalosauripus } \\ \text { Moraesauichnus } & \text { Stegopodus } & \text { Therangospodus } \\ \text { Trisauropodiscus } & \text { Wildeichnus } & \end{array}$

\section{Pterosaurios: Agadirichnus, Pteraichnus.}

\section{Anápsidos: Emydhipus.}

4. Cocodrilos: Hatcherichnus, Crocodylopodus.

5. Otros dinosaurios no clasificables a nivel icnogenérico.

En Marruecos se definieron:

- Agadirichnus elegans Ambroggi y De Lapparent, 1954 , las primeras huellas descubiertas de pterosaurio;

- Breviparopus taghbaloutensis Dutuit y Ouazzou,
1980, que estableció una base de clasificación de las huellas saurópodas;

- Boutakioutichnium atlasicus Nouri, Díaz-Martínez y Pérez-Lorente, 2011, de anatomía anormal de los huesos metatarsianos.

En Marruecos están los únicos ejemplares africanos de Otozoum y Macropodosaurus. Este último solo se conocía en tres yacimientos (Asia, Europa y América del Norte). De la misma manera fue el país donde se describió la huella (90 cm de largo) del mayor dinosaurio carnívoro conocido, que, con interrupciones, pisó en un trayecto de unos $2 \mathrm{~km}$ (louaridène). También hay rastrilladas (secuencias continuas de pisadas de un animal) excepcionalmente largas o con muchas huellas (80 pisadas en Adrar-Ougladal) que visualizan bastante tiempo de actividad y gran espacio atravesado. En ellas se deduce: cojera, lateralidad, corrección de rumbo, variabilidad de los morfotipos y el estado del suelo. Hay yacimientos con varios tipos de dinosaurios (Aït Arbi) y otros con grupos monoespecíficos o manadas (saurópodos en Imi-n-Tanout). Ciertos dinosaurios eligen el tipo de marcha según las condiciones del suelo: al SE de louaridène predomina la marcha semiplantígrada (apoyan el metatarso) mientras que al NO predomina la digitígrada. 


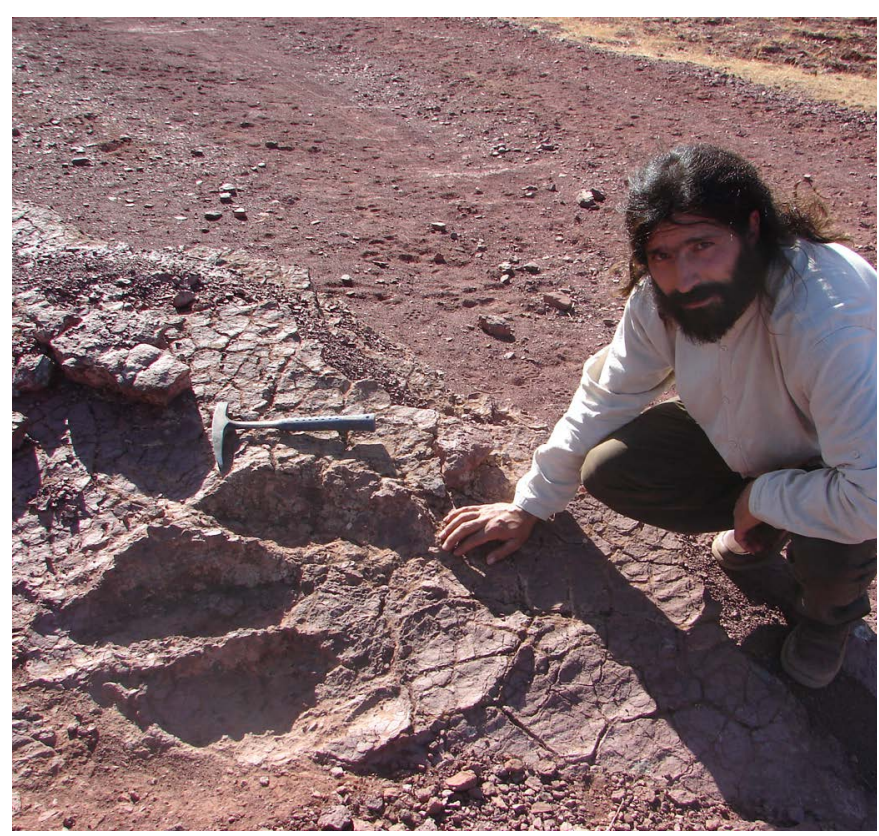

Huellas grandes de louaridène | foto Félix Pérez-Lorente

Plateau, Rouch y Giboulet publicaron en 1937 el primer trabajo sobre huellas de dinosaurio marroquíes. Entre 1937 y 1980 se publicaron seis; y siete, entre 1980 y 2000. Desde el año 2000 se han publicado unos 50 trabajos y se han leído dos tesis doctorales sobre icnitas, una en la Universidad de Rabat. Actualmente investigan universidades de seis países en colaboración con las de Agadir, El Jadida, Marrakesh, Oujda y Rabat. Hay que destacar a la Asociación para la Protección del Patrimonio Paleontológico de Marruecos en investigación y divulgación. Por su parte, el Geoparque M'Goum, reconocido por la UNESCO desde 2014, situado en el Alto Atlas Central de Azilal-Demnate, es un conjunto patrimonial basado en los yacimientos con huellas de dinosaurio.

A pesar de la constancia de tanta riqueza patrimonial en la zona, existen problemas de conservación de los yacimientos marroquíes. Los principales son:

$>$ La meteorización, la metaestabilidad de aforamientos en taludes y, en Anza, la erosión marina. Se han hecho campañas anuales de restauración (2007-2012) con alumnos dirigidos por especialistas, en Iouaridène, Aït Blal e Ibaqualliwn.

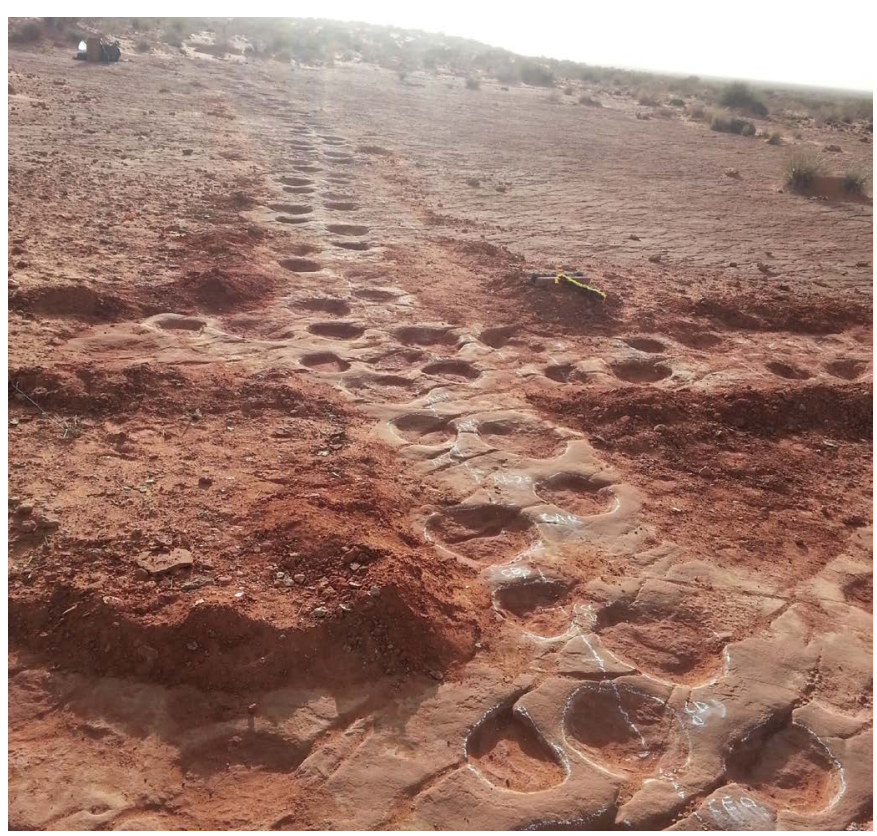

Rastrillada saurópoda de Jarrate Lbel | foto Moussa Masrour

$>$ Las canteras para extraer roca. Algunos yacimientos marroquíes han aflorado por este sistema de explotación (Ifgh) y puede que desaparezcan por el mismo procedimiento.

$>$ El desconocimiento: vandalismo, destrucción en el borde de parcelas cultivadas, paso de ganado o de vehículos por encima de las superficies con huellas

$>$ La falta de coordinación entre organismos públicos condujo a la destrucción de parte de Ouzanwrou en louaridène. Hay que señalar también lo contrario en el caso de la carretera que pasa por el yacimiento de Aït Blal desviada para no afectarlo.

Las huellas de dinosaurio de Marruecos son patrimonio natural, cultural y científico. Su interés se muestra por el aumento de los estudios y publicaciones. Su conservación depende de las medidas de protección que se tomen, fundamentalmente de educación de la población. 